\title{
The Association Between the Use of Statins and Clinical Outcomes in Patients with COVID-19: A Systematic Review and Meta-analysis
}

\author{
Chia Siang Kow ${ }^{1,4} \cdot$ Syed Shahzad Hasan ${ }^{2,3}$ \\ Accepted: 27 June 2021 / Published online: 3 August 2021 \\ (c) The Author(s), under exclusive licence to Springer Nature Switzerland AG 2021
}

\begin{abstract}
Purpose Previously, we have reported potential clinical benefits with the use of statins in patients with coronavirus disease 2019 (COVID-19) in a meta-analysis, where there was a significantly reduced hazard for a fatal or severe course of illness with the use of statins, but the meta-analysis was limited by the small number of studies included, with small heterogeneity among studies, due to the unavailability of more studies at the point of literature search. We aimed to perform an updated systematic review and meta-analysis to summarize the existing evidence on the effect of statins on the clinical outcomes of patients with COVID-19.

Methods Electronic databases, including PubMed, Google Scholar, and Scopus, and preprint servers were searched (last updated June 3, 2021) to identify studies investigating the association between the use of statins in patients with COVID-19 and the development of severe disease and/or mortality. Random-effects model meta-analyses were performed to estimate the pooled odds ratio (OR) or hazard ratio (HR) with $95 \%$ confidence intervals (CIs). The outcomes of interest were (1) allcause mortality and (2) a composite endpoint of severe illness of COVID-19.

Results Upon systematic literature search, we identified 35 studies, of which 32 studies reported the outcome of all-cause mortality and 15 studies reported the composite endpoint of severe COVID-19 illness between statin users versus non-statin users with COVID-19. Our meta-analysis revealed that the use of statins was associated with a significantly lower risks of all-cause mortality ( $\mathrm{HR}=0.70,95 \%$ CI $0.58-0.84, n=21,127$, and $\mathrm{OR}=0.63,95 \%$ CI $0.51-0.79, n=115,097)$ and the composite endpoint of severe illness ( $\mathrm{OR}=0.80,95 \% \mathrm{CI} 0.73-0.88, n=10,081)$ in patients with COVID-19, compared to non-use of statins, at the current sample size.

Conclusion Statin use is associated with a better prognosis in patients with COVID-19. Our findings provide a rationale to investigate the use of statins among patients with COVID-19 in large scale clinical trials.
\end{abstract}

\section{Introduction}

The global spread of the coronavirus disease 2019 (COVID-19) pandemic has called for the development of novel pharmacological agents or repurposing of existing

Chia Siang Kow

chiasiang_93@hotmail.com

1 School of Postgraduate Studies, International Medical University, Kuala Lumpur, Malaysia

2 School of Applied Sciences, University of Huddersfield, Huddersfield, United Kingdom

3 School of Biomedical Sciences and Pharmacy, University of Newcastle, Callaghan, Australia

4 School of Pharmacy, Monash University Malaysia, Bandar Sunway, Petaling Jaya, Selangor, Malaysia

\section{Key Points}

Establishing the clinical benefits of in-hospital or preadmission use of statins in patients with coronavirus disease 2019 (COVID-19) would have significant clinical implications; where statin treatment is appropriately indicated, clinicians should ensure patients are receiving statins with appropriate intensity.

The use of statins was associated with a significantly lower risk of all-cause mortality and the composite endpoint of severe illness in patients with COVID-19 compared to non-use of statins. 
pharmacological agents to prevent disease progression and deaths in patients with COVID-19. Previously, we have reported potential clinical benefits with the use of statins in patients with COVID-19, where our meta-analysis of four observational studies with over 9000 patients with COVID-19 revealed a significantly reduced hazard for a fatal or severe course of illness with the use of statins compared to non-use of statins [pooled hazard ratio (HR) $=0.70 ; 95 \%$ confidence interval (CI) 0.53-0.94] [1]. However, our metaanalysis on the effect of statins in patients with COVID19 was limited by the small number of studies included, and with small heterogeneity among studies, due to the unavailability of more studies at the point of literature search.

Establishing the clinical benefits of in-hospital or preadmission use of statins in patients with COVID-19 would have significant clinical implications, not only in the management of patients with COVID-19, but also in the management of patients at high cardioascular risk of or with established cardiovascular disease, who have not acquired COVID-19 as yet, since it would provide another rationale for clinicians to ensure that patients in this population, where statin treatment is appropriately indicated, are receiving a statin with appropriate intensity, particularly amid the COVID-19 pandemic [2, 3]. In terms of the management of patients with COVID-19, we have thus far struggled to achieve a significant breakthrough to discover life-saving therapeutic interventions, except for systemic corticosteroids, with the latest announcement of findings from the World Health Organization's Solidarity Trial reporting a failure to show clinical benefits with the use of the muchhyped remdesivir and other antiviral agents $[4,5]$. Therefore, if statin treatment could be proven to provide clinical benefits in patients with COVID-19, the lives of hundreds of thousands could be saved, since statins are readily available and are one of the most prescribed drugs worldwide [6].

Since the publication of our meta-analysis [1] on the effect of statins in patients with COVID-19, a few [7-9] have commented on the reliability of our findings. With more studies available, we aimed to perform an updated systematic review and meta-analysis to determine the overall effect of statins on the clinical outcomes of patients with COVID19. In this article, we also discuss the comments [7-9] to our previous meta-analysis [1].

\section{Methods}

\subsection{Search Strategy and Selection Criteria}

The eligibility criteria for inclusion of studies in this systematic review included (1) original research that investigated the association between the use of statins, regardless of type, dose, and duration, in patients with COVID-19 and the development of severe disease and/or mortality; (2) cohort studies and case-control studies that provided measurement of the association with adjustment of confounders; and (3) studies published in any language and with any sample size. Exclusion criteria included (1) commentaries, editorials, narrative reviews, in vitro and in vivo studies, and other irrelevant study designs and (2) studies that provided measurement of the association without adjustment of confounders.

We performed a systematic literature search in electronic databases, including PubMed, Google Scholar, Scopus, and preprint servers (medRxiv, Research Square, SSRN). We also hand-searched the reference lists of relevant reviews and included studies. We last updated our literature search on June 3, 2021. The search strategy was built based on the following keywords and their Medical Subject Headings (MeSH) terms: "COVID-19," "statin," and "HMG-CoA reductase." Two investigators (CSK and SSH) independently performed literature screening to identify eligible studies, and disagreements were resolved through mutual discussion.

\subsection{Data Extraction and Risk of Bias Assessment}

The data extraction and risk of bias assessment were performed by the first investigator (CSK) and cross-checked by the second investigator ( $\mathrm{SSH}$ ). Data extracted from the included studies included the name of the first author, study design, study location, total number of patients, patients' age, type of statins received, event numbers, and summary estimates of effect measures, including adjusted odds ratios (aORs) and adjusted hazard ratios (aHRs). The quality of observational studies was evaluated using the Newcastle-Ottawa Scale [10].

The outcomes of interest were (1) all-cause mortality and (2) a composite endpoint of severe illness of COVID-19, which included the requirement of intubation/mechanical ventilation and/or admission into an intensive care unit, and being categorized as a severe/critical course of illness as defined by the authors. The definition of severe/critical disease varied across the included studies.

\subsection{Data Analysis}

The pooled OR or pooled HR with $95 \%$ CIs was used as a summary relative effect measure. The random-effects model was used to perform the meta-analysis given the potential heterogeneity across the included studies. The statistical heterogeneity was determined using the $I^{2}$ and $Q$ statistics. Subgroup analyses were conducted to determine potential differences based on the regions where the included studies were performed. Sensitivity analyses were conducted to evaluate the robustness of the results by limiting to studies that included patients with laboratory-confirmed COVID-19 
(instead of clinically or radiologically confirmed COVID19), studies that confirmed the continuation of statin therapy during hospitalization for COVID-19, and studies in which the definition of severe illness is based on the requirement of mechanical ventilation or intubation. A funnel plot for asymmetry was visually inspected to evaluate the included studies for publication bias. All the analyses were performed using Meta XL, version 5.3 (EpiGear International, Queensland, Australia).

\section{Results}

\subsection{Study Selection and Characteristics}

We retrieved 965 records from the combination of two independent searches. After the removal of duplications and irrelevant literature, 56 full-text articles were assessed for eligibility. A total of 35 studies [11-45] (32 published studies [11-24, 26-29, 31-35, 37-45] and three studies available as a preprint $[25,30,36])$ that corresponded to the inclusion and exclusion criteria were included in the systematic review and meta-analyses (Fig. 1).

All the included studies [11-45] are observational studies, reported as cohort/case-control studies or database reviews. All except seven studies [18, 26, 29, 34, 35, 38, 39] were based directly on the hospital (or nursing home) medical records, and 12 of the included studies $[13,20,22,23,25$, $31,33,36,41-44]$ are single-centered observational studies. These hospital- (or nursing home)-based studies [11-17, 19-25, 27, 28, 30-34, 36, 37, 40-45] covered a moderate number of participants per study (median 840.0, interquartile range 286.5-1997.5). The remaining seven studies [18, $26,29,34,35,38,39]$ analyzed data from the nation-level registries (retrospective database reviews), and included a range of 1868-64,781 participants. The regions where the included studies were performed span Asia (China $[n=4]$ [11, 12, 27, 42], Iran [ $n=1]$ [31], and Korea [ $n=1]$ [39]), Europe (Italy $[n=4][14,23,28,37]$, the United Kingdom $[n=2][25,30]$, Belgium $[n=1]$ [15], France [ $n=1]$ [19], Spain $[n=3][24,35,40]$, Denmark [ $n=1]$ [26], Poland [ $n=1$ ] [44], Sweden [ $n=1$ ] [38], and Belgium [ $n=1$ ] [32]), and North America (the United States of America [ $n$ $=14][13,16-18,20-22,29,33,34,36,41,43,45])$. Seven studies [15, 19, 32-34, 38, 42] (could have) included both laboratory-confirmed and clinically or radiologically confirmed patients with COVID-19, while the remaining studies [11-14, 16-18, 20-31, 33-37, 39-41, 43-45] included only laboratory-confirmed patients with COVID-19. Eleven studies $[12,13,18,20,27,32,35,36,39,42,44]$ confirmed the continuation/de novo initiation of statins during hospitalization for COVID-19. Table 1 summarizes the main characteristics of all the included studies.
Fig. 1 Flow diagram of the study selection

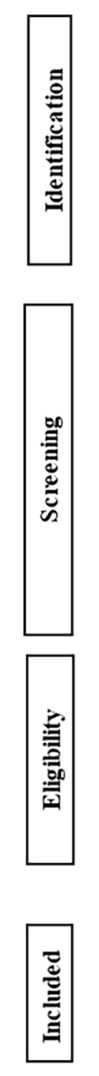

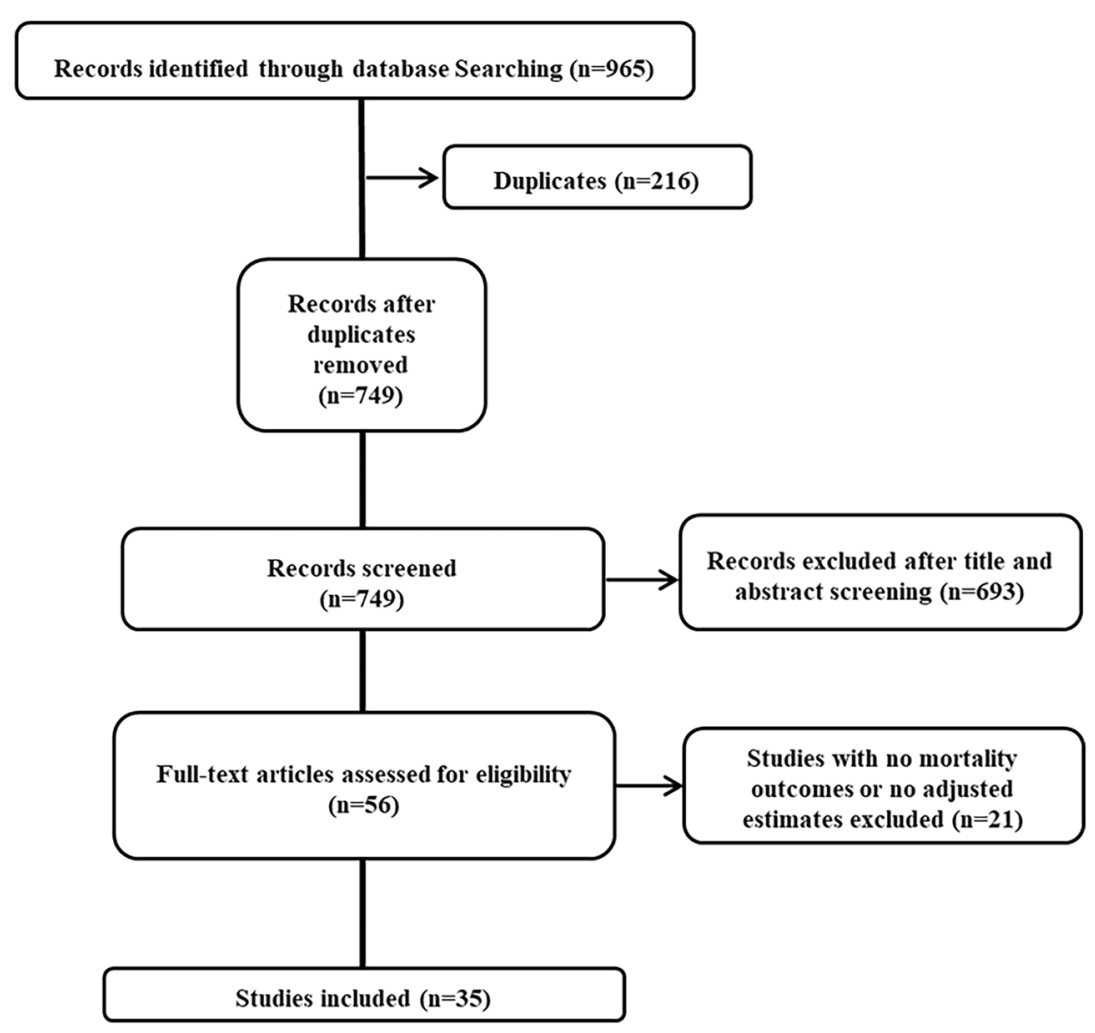


Assessment with the Newcastle-Ottawa Scale revealed that other than three studies $[11,19,20]$, which are of moderate quality (5/9 to 6/9), all studies [12-18, 21-45] are of good quality (at least 7/9). None of the included studies are of poor quality.

\subsection{Use of Statins and All-Cause Mortality in Patients with COVID-19}

The outcome of all-cause mortality is available in 32 studies [12-14, 16-21, 23-45] comparing statin users versus nonstatin users with COVID-19, of which ten studies [12-14, $20,24,26,27,31,36,45]$ provided adjusted measure as HR, whereas 22 studies [16-19, 21, 23, 25, 28-30, 32-35, 37-44] provided adjusted measure as OR. The meta-analysis of studies with an HR, which included 21,127 patients with COVID-19, revealed that the use of statins was associated with a significantly lower hazard of all-cause mortality in patients with COVID-19 compared to non-use of statins (Fig. 2B; HR =0.70; 95\% CI 0.58-0.84). The pooled OR is consistent with the pooled HR, which also demonstrated significantly reduced odds of all-cause mortality with the use of statins in patients with COVID-19 compared to nonuse of statins (Fig. 2A; OR $=0.63 ; 95 \%$ CI 0.51-0.79; $n=115,097$ ). Visual inspection of the funnel plot (Fig. 4A) identified some degree of asymmetry.

Subgroup analyses with studies originating from Asia $(\mathrm{HR}=0.56 ; 95 \%$ CI 0.42-0.75) $[12,27,31]$, Europe $(\mathrm{OR}=$ 0.77; 95\% CI 0.64-0.94) [19, 23, 25, 28, 30, 32, 35, 37, 38, $40,44]$, and North America (OR $=0.62 ; 95 \%$ CI 0.54-0.73), respectively [16-18, 21, 29, 33, 34, 41, 43], observed significantly reduced risks of mortality with the use of statins in patients with COVID-19 compared to non-use of statins. Findings from the sensitivity analyses with studies [12-14, 16-18, 20, 21, 23-31, 35-37, 39-41, 43-45] including only laboratory-confirmed patients with COVID-19 (OR = 0.67; 95\% CI 0.56-0.79 and HR $=0.70 ; 95 \%$ CI 0.58-0.84) and studies $[12,13,20,27,32,35,36,39,42$, 44] that confirmed the continuation of statins during hospitalization for COVID-19 (OR =0.61; 95\% CI 0.52-0.73 and HR =0.59; 95\% CI 0.41-0.84), are consistent with the main analyses.

\subsection{Use of Statins and Development of Severe IIIness in Patients with COVID-19}

A total of 15 studies $[11,12,15-17,19,22,26-28,31$, $35-37,43]$ reported the composite endpoint of severe illness of COVID-19, and all except four studies [12, 26, 27, 36] provided adjusted measure as OR. The definition of severe illness varied across the 15 studies: in the study by Yan et al. [11], the definition is based on that given in the Diagnosis and Treatment Protocol for Novel Coronavirus Pneumonia by the Chinese National Health Commission; in the studies by Zhang et al. [12], Butt et al. [26], Fan et al. [27], Mitacchione et al. [28], and Greco et al. [37] respectively, the definition is based on admission into an intensive care unit or pulmonology department; in the study by De Spiegeleer et al. [15], the definition is based on long-stay hospital admission or death; in the studies by Gupta et al. [16], Cariou et al. [19], and Peymani et al. [31] respectively, the definition is based on the requirement of intubation/ mechanical ventilation; in the studies by Song et al. [17] and Lohia et al. [43] respectively, the definition is based on admission into an intensive care unit or the requirement of intubation; in the studies by Daniels et al. [22] and Memel et al. [36] respectively, the definition is based on admission into an intensive care unit or death; in the study by TorresPeña et al. [35], the definition is based on development of acute respiratory distress syndrome.

The meta-analysis of studies $[11,15-17,19,22,28,31$, $35,37,43$ ] with ORs, which altogether included 10,081 patients with COVID-19, observed that the use of statins was associated with significantly lower odds of development of severe illness in patients with COVID-19 compared to nonuse of statins (Fig. 3A; OR $=0.80 ; 95 \%$ CI $0.73-0.88$ ). The pooled HR is consistent with the pooled OR $(n=10,738)$, which demonstrated a non-significantly reduced hazard of development of severe illness with the use of statins in patients with COVID-19 compared to non-use of statins (Fig. 3B; HR =0.80; 95\% CI 0.56-1.14). Visual inspection of the funnel plot (Fig. 4B) identified some degree of asymmetry.

Subgroup analyses with studies originating from Europe $(\mathrm{OR}=0.79 ; 95 \%$ CI 0.71-0.89) $[15,19,28,35,37]$ and North America $(\mathrm{OR}=0.79 ; 95 \%$ CI $0.59-1.05)$, respectively $[16,17,22,43]$, observed reduced risks of mortality with the use of statins in patients with COVID-19 compared to nonuse of statins. Sensitivity analyses with studies [11, 16, 17, $22,28,31,35,37,43$ ] including only laboratory-confirmed patients with COVID-19 (OR $=0.79 ; 95 \%$ CI 0.72-0.88), studies $[12,27,36]$ that confirmed the continuation of statins during hospitalization for COVID-19 (HR $=0.71 ; 95 \% \mathrm{CI}$ 0.55-0.92), and studies in which the definition of severe illness is based on the requirement of mechanical ventilation or intubation $[16,17,19,31,43](\mathrm{OR}=0.81 ; 95 \% \mathrm{CI}$ 0.69-0.96) also revealed consistent findings with the main analyses.

\section{Discussion}

Before the publication of our first meta-analysis [1] on the effect of statins in patients with COVID-19, the use of statins had already gained attention; some hypothesized that statins might assume clinical benefits in patients with COVID-19 based on their several mechanisms of action [45, 46], and yet 


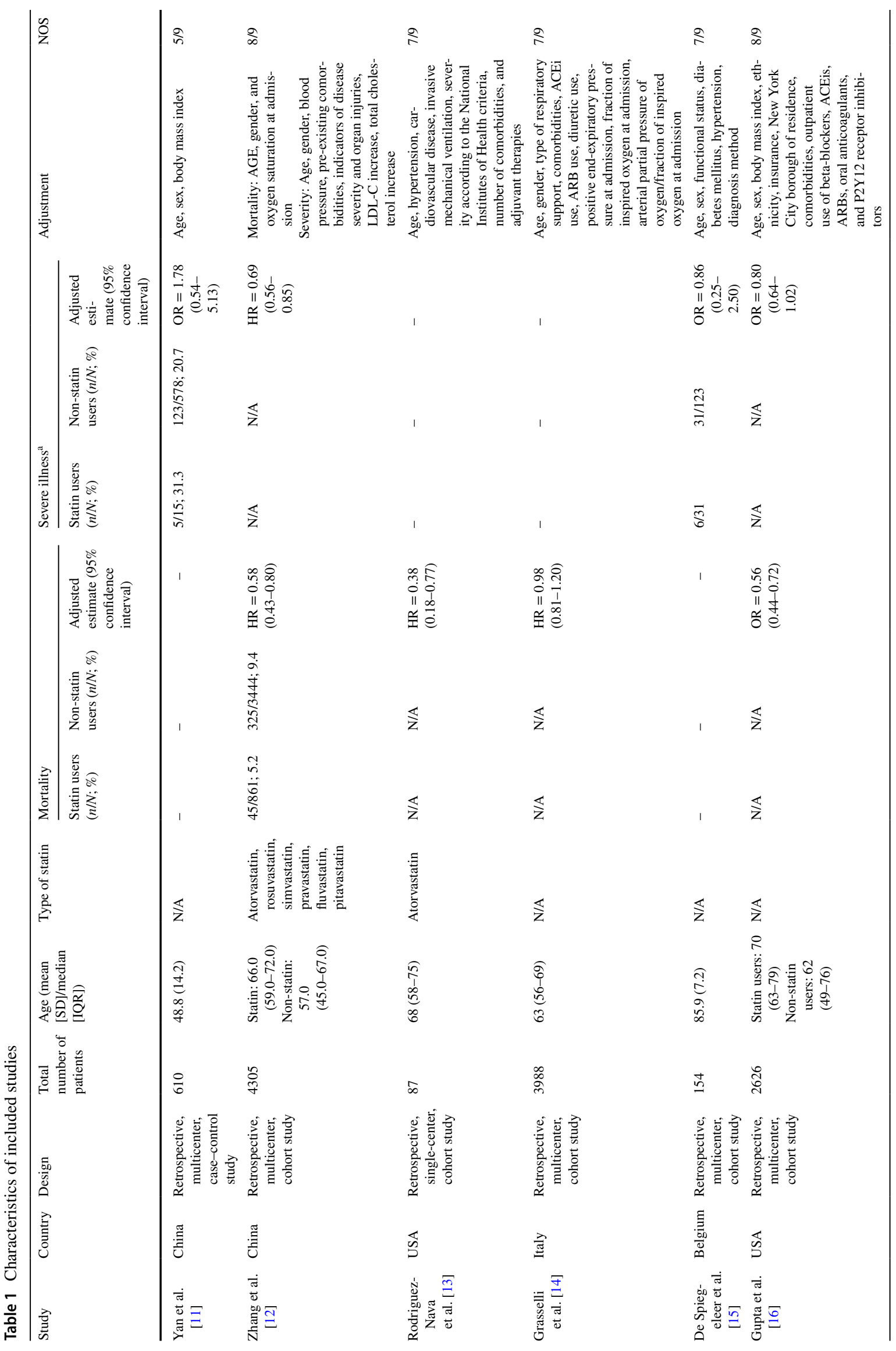




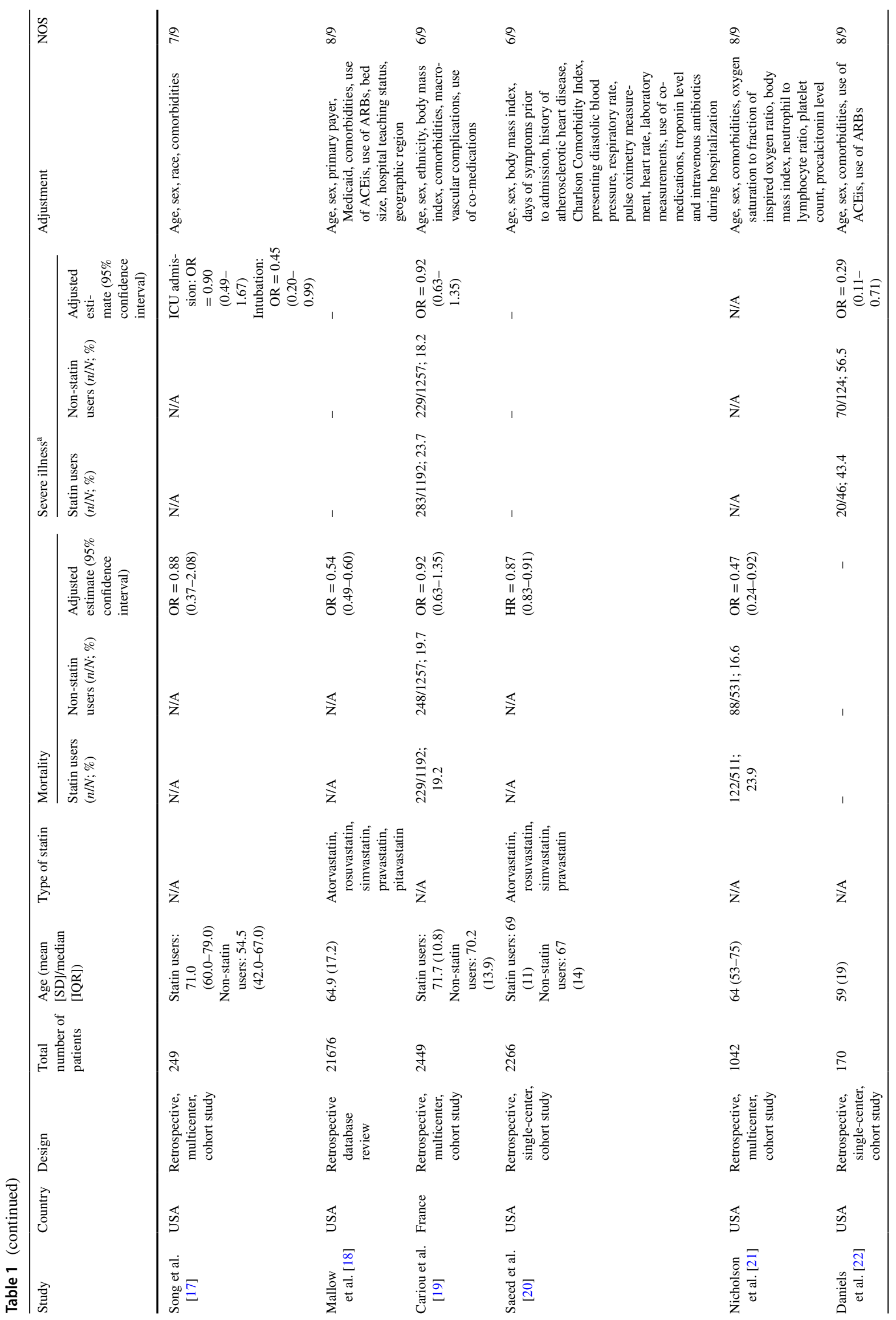




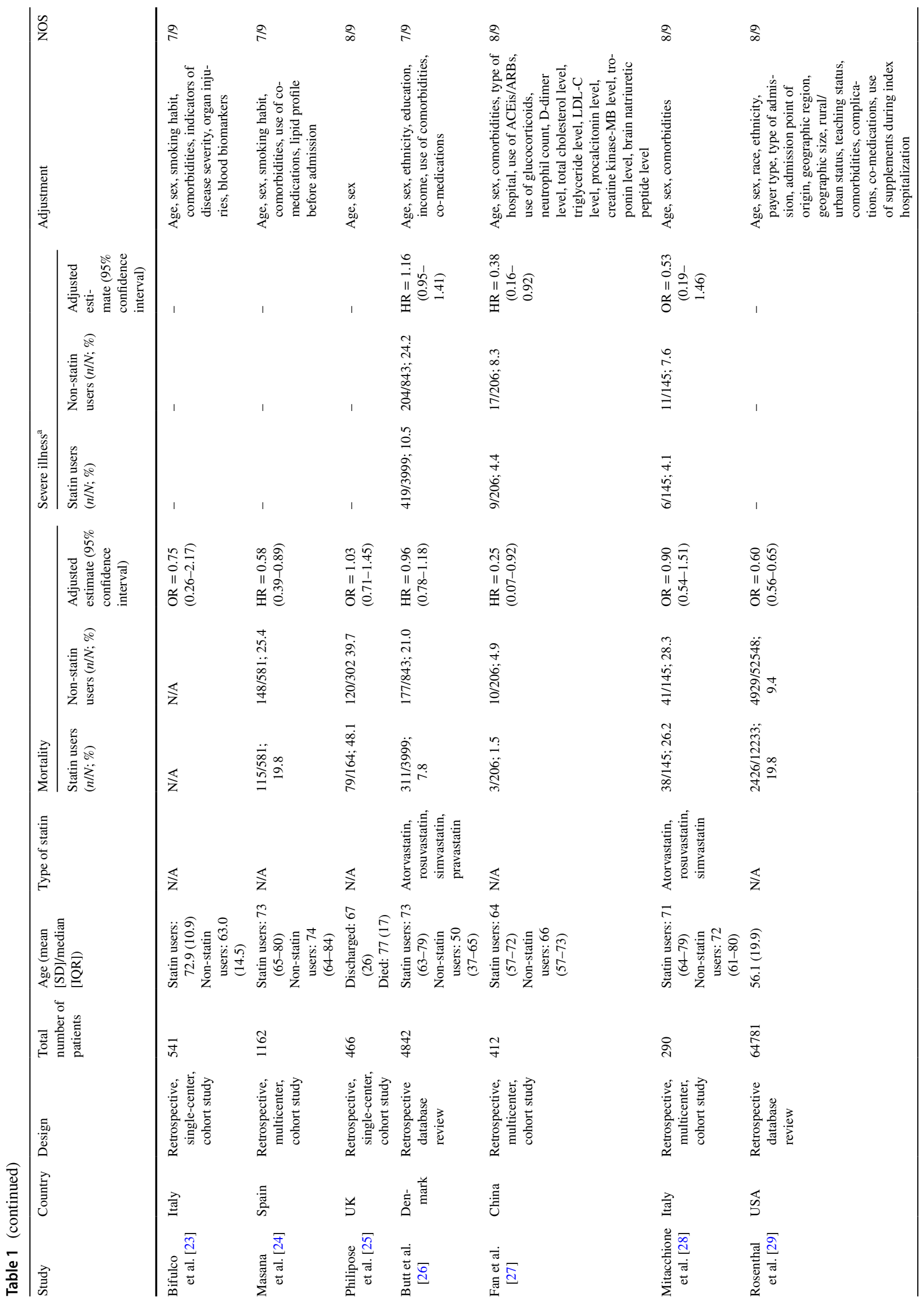




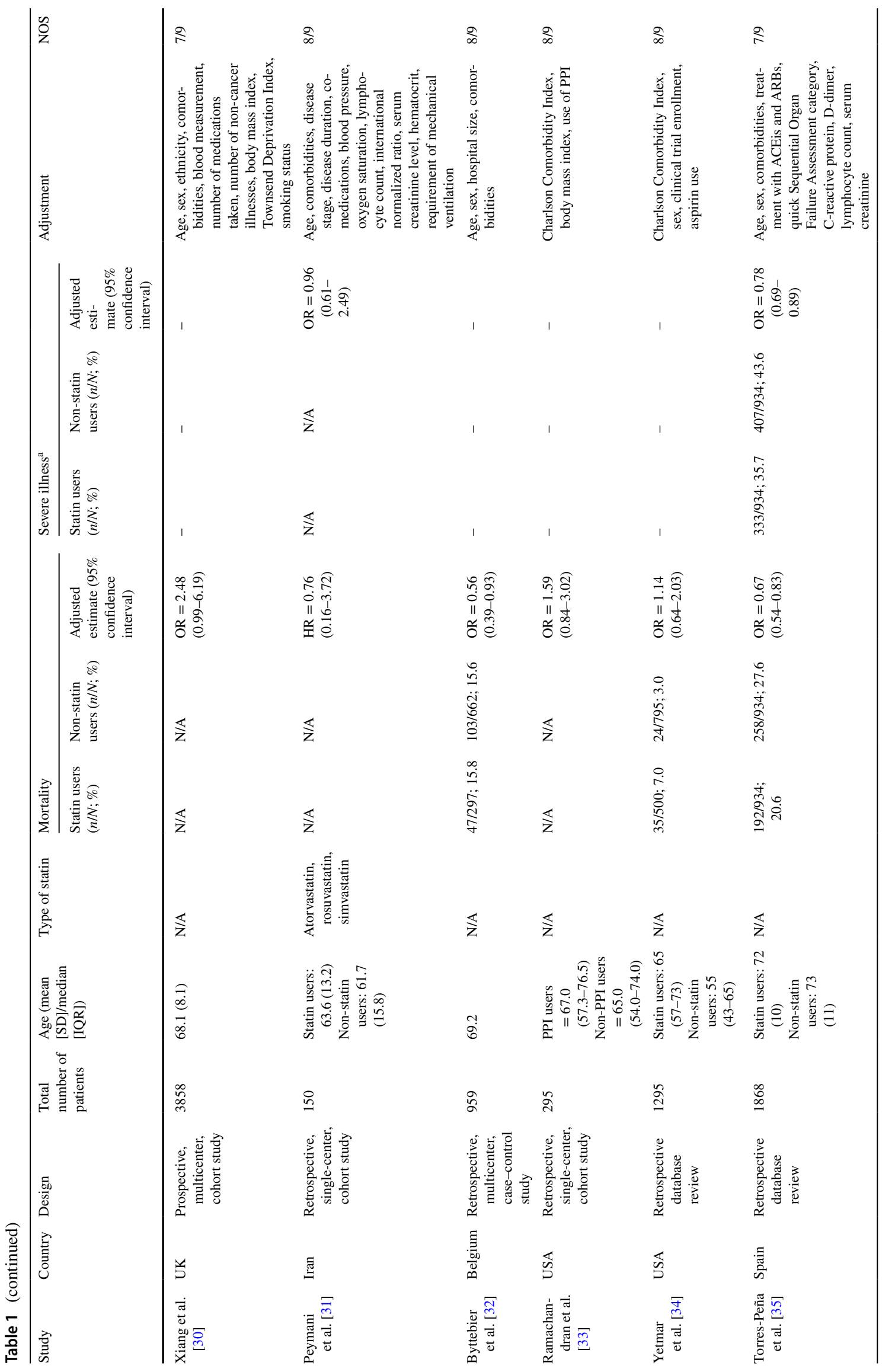




\begin{tabular}{|c|c|c|c|c|c|c|c|c|c|}
\hline \multicolumn{2}{|c|}{ ్ֶ } & $\stackrel{s}{i}$ & $\stackrel{\widehat{\partial}}{\infty}$ & $\stackrel{s}{r}$ & $\stackrel{\Omega}{\Sigma}$ & $\stackrel{\curvearrowright}{\infty}$ & $\stackrel{\curvearrowright}{\infty}$ & sे & $\stackrel{\partial}{\infty}$ \\
\hline \multicolumn{2}{|c|}{ 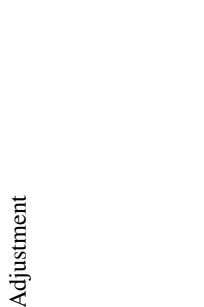 } & 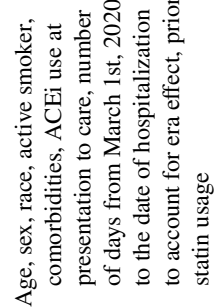 & 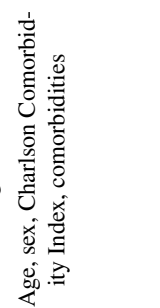 & 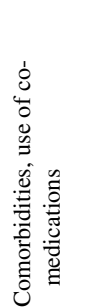 & 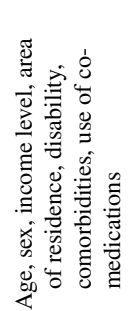 & 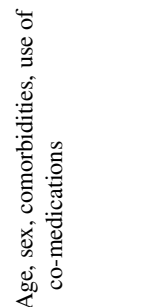 & 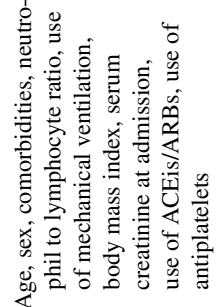 & 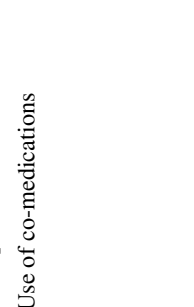 & 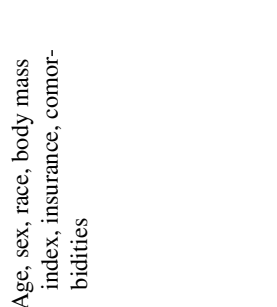 \\
\hline \multirow[b]{3}{*}{ 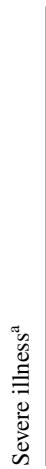 } & 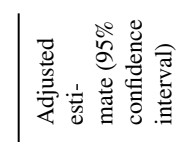 & 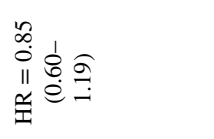 & 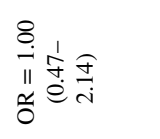 & 1 & 1 & 1 & 1 & 1 & 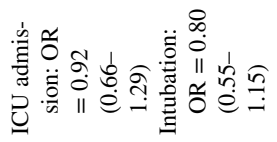 \\
\hline & 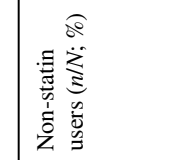 & $\overleftrightarrow{\mathrm{z}}$ & $\begin{array}{l}\vec{a} \\
i \\
\ddot{n} \\
\stackrel{y}{m} \\
\ddot{z}\end{array}$ & 1 & 1 & 1 & 1 & 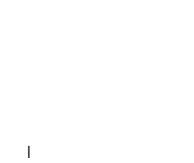 & 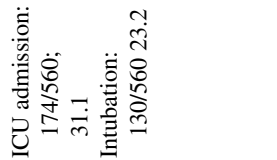 \\
\hline & 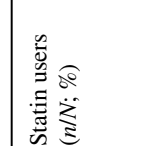 & $\overleftrightarrow{\mathrm{z}}$ & 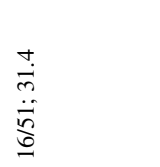 & 1 & 1 & 1 & 1 & 1 & 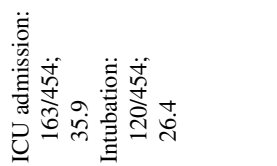 \\
\hline & 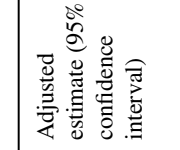 & 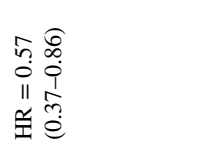 & 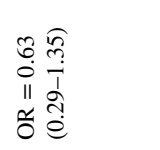 & 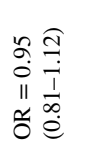 & 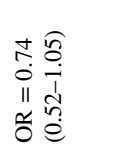 & 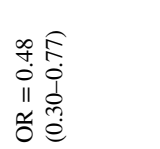 & 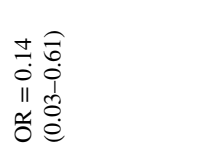 & 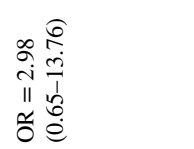 & 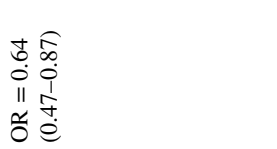 \\
\hline & 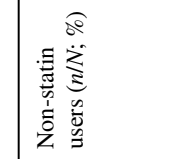 & 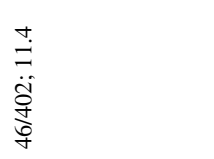 & $\begin{array}{l}\exists \\
\bar{m} \\
\dot{0} \\
\text { on } \\
\text { 亲 }\end{array}$ & 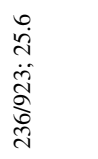 & $\overleftrightarrow{\mathrm{z}}$ & 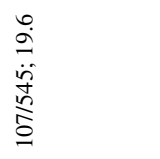 & 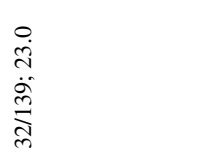 & $\overleftrightarrow{z}$ & 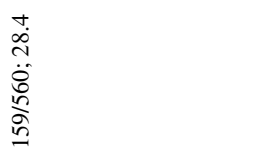 \\
\hline & 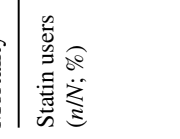 & 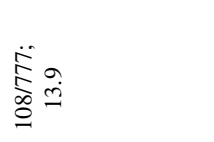 & 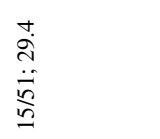 & 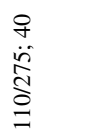 & $\overleftrightarrow{\mathrm{z}}$ & 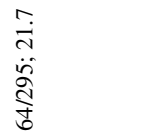 & $\begin{array}{l}\vec{\infty} \\
\stackrel{\infty}{\ddot{*}} \\
\stackrel{\vec{N}}{\Xi}\end{array}$ & $\overleftrightarrow{\mathrm{z}}$ & 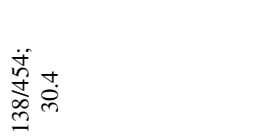 \\
\hline & & 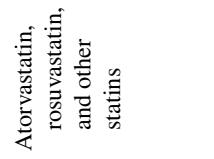 & $\mathbb{z}$ & $\overleftrightarrow{\mathbf{z}}$ & $\widehat{\underline{z}}$ & $\widehat{্}$ & $\widehat{\underline{z}}$ & $\mathbb{Z}$ & 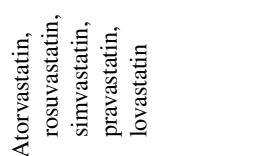 \\
\hline & 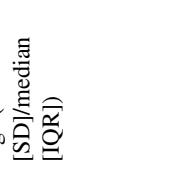 & 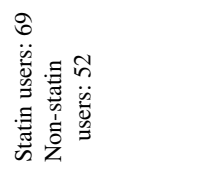 & 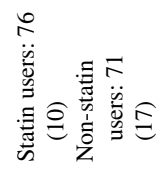 & $\begin{array}{l}\hat{0} \\
i \\
i=6 \\
b \\
0\end{array}$ & $\overleftrightarrow{\mathbb{Z}}$ & 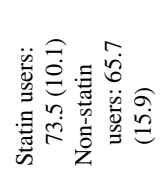 & 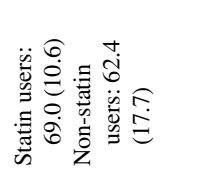 & 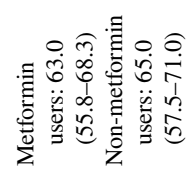 & 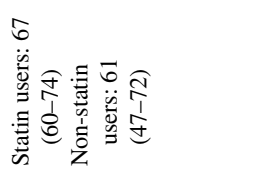 \\
\hline & 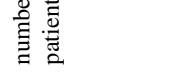 & $\stackrel{\vartheta}{\Xi}$ & 家 & 志 & $\stackrel{\infty}{\stackrel{D}{\curvearrowright}}$ & 承 & 号 & $\stackrel{\mathscr{N}}{\sim}$ & $\stackrel{t}{\Xi}$ \\
\hline & & 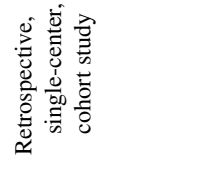 & 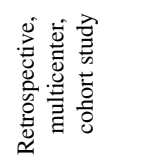 & 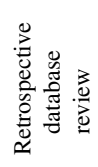 & 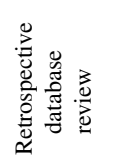 & 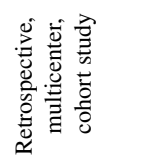 & 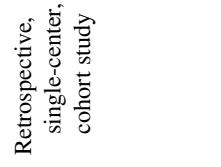 & 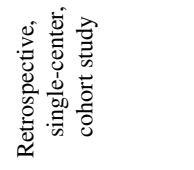 & 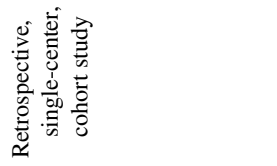 \\
\hline & & $\overleftrightarrow{\Delta}$ & त्ञ્ज & $\begin{array}{l}\text { 丞 } \\
\overline{\tilde{s}}\end{array}$ & : & $\begin{array}{l}\text { 量 } \\
\text { की }\end{array}$ & 峁 & 㺃 & $\overleftrightarrow{\Delta}$ \\
\hline & & 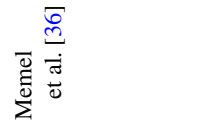 & 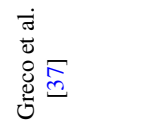 & 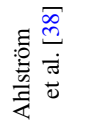 & 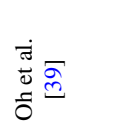 & 守 & 量 & 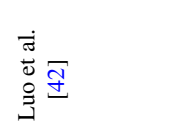 & 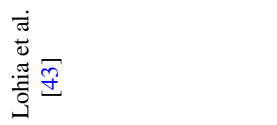 \\
\hline
\end{tabular}




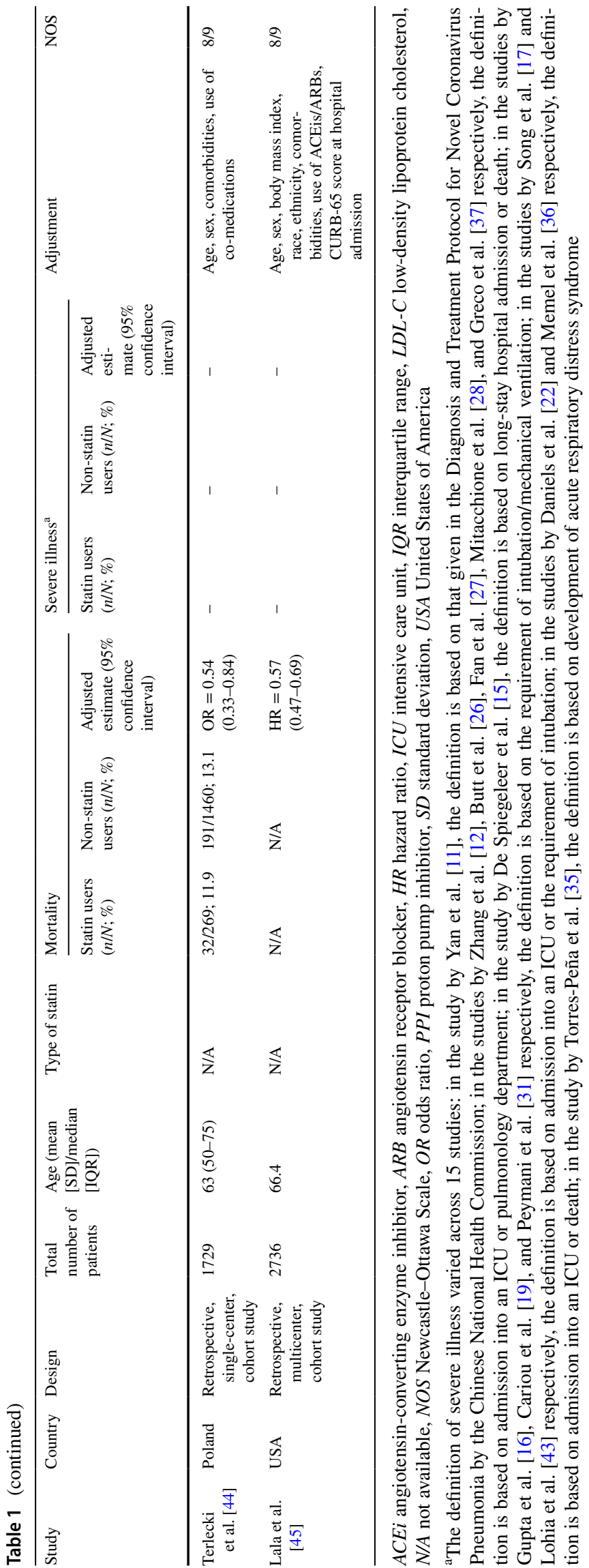

some postulated that statins might cause harms in patients with COVID-19, citing a heightened risk for the development of acute respiratory distress syndrome, amongst others [47-49]. We have in our meta-analysis [1] denied the possibility of harms with the use of statins in patients with COVID-19, and again, in our updated meta-analysis with more studies and a larger cohort of patients included, we substantiated our previous findings where the use of statins could reduce the risk of development of a fatal course or a severe course of illness in patients with COVID-19.

Our findings that statins were beneficial to patients with COVID-19 had their biological plausibility. Nevertheless, the mechanisms by which statins exert beneficial effects are still not known with certainty due to their pleiotropic effects: statins could modulate inflammation and immune response, as well as exert direct antiviral effects, and improve endothelial function. It has been demonstrated that statins can inhibit the activity of transcription factors, including activator protein-1 and nuclear factor kappa-light-chain-enhancer of activated B cells (NF-KB), which are involved in mediating inflammatory pathways of coronavirus infection such as interleukin- $1 \beta$ signaling [50]. In addition, statin therapy has been shown to downregulate the expression of toll-like receptor- 4 on immune cells, with subsequent downregulation of NF-KB activity, and, therefore, a decrease in the secretion of inflammatory cytokines and a reduced risk for the development of acute distress respiratory syndrome [51].

On the other hand, statins' direct antiviral effect involves a reduction in the percentage of cholesterol present in the plasma membrane and subsequent alteration of the assembly of viral receptors, thereby markedly reducing the possibility of entry of coronaviruses into host cells, since coronaviruses bind specific receptors (angiotensin-converting enzyme 2) that are concentrated at the areas of the plasma membrane rich in cholesterol, also known as lipid rafts [52, 53]. Furthermore, potential improvement in endothelial function by statins among patients with COVID-19 could be achieved through reduced platelet aggregation, increased thrombomodulin expression on endothelial cells, and decreased tissue factor expression [54]. This mechanism of statins is highly desirable since endothelial dysfunction is the common denominator of a range of clinical manifestations of a severe course of COVID-19 [55].

There are concerns that the use of statins in patients with COVID-19 may heighten the risk and severity of viral-induced myopathies. However, clinically significant myonecrosis with an elevation of serum level of creatine kinase more than ten times normal in conjunction with muscle symptoms developed in less than $0.5 \%$ of patients receiving statin therapy in large-scale clinical trials [56-59]. Moreover, clinical rhabdomyolysis, defined as myonecrosis with myoglobinuria or acute renal failure, continues to be a very rare event during statin therapy, with an incidence of 
hospitalization for rhabdomyolysis about 0.44 per 10,000 patient-years [60]. While COVID-19-associated myositis is likely not a frequent clinical manifestation, patients with symptomatic or asymptomatic myonecrosis or clinical rhabdomyolysis should discontinue statin therapy immediately.

Since statins could assume benefits in patients with COVID-19, the dosing of statin therapy for the treatment of COVID-19 has become an important issue. Only two included studies $[12,13]$ revealed the dosing regimen of statins received by the patients; Zhang et al. [12] reported a median atorvastatin equivalent dose of $20 \mathrm{mg} /$ day, whereas Rodriguez-Nava et al. [13] reported the use of atorvastatin at a dose of $40 \mathrm{mg} / \mathrm{day}$. Nevertheless, there have been three studies $[26,28,43]$ that evaluated the difference in outcomes between low-/moderate-intensity statin therapy and highintensity therapy. Butt et al. [26] reported no significant difference in the risk of all-cause mortality ( $\mathrm{HR}=1.07$; $95 \%$ CI $0.77-1.50)$ and the risk of severe disease (HR $=0.81$; 95\% CI 0.60-1.10) in patients with COVID-19 who received low-/moderate-intensity statin therapy compared to those who received high-intensity statin therapy. Similarly, Mitacchione et al. [28] reported no significant difference in the risk of in-hospital mortality $(\mathrm{OR}=1.17$; 95\% CI $0.77-1.76)$ in patients with COVID-19 who received moderate-intensity statin therapy compared to their counterparts who received high-intensity statin therapy. In contrast, the study by Lohia et al. [43] revealed that moderate-intensity and high-intensity but not low-intensity statin therapy was associated with a significantly lower risk of mortality $(\mathrm{OR}=0.52 ; 95 \% \mathrm{CI}$ $0.31-0.87$ and $\mathrm{OR}=0.54 ; 95 \%$ CI $0.29-0.99$ ) compared to no statin therapy, among propensity-matched patients with COVID-19.

Fedson [7] commented that we had in our previous meta-analysis considered studies regardless of whether the included patients received statin therapy either during hospitalization or as outpatients. Therefore, our estimate of the effect of statins was probably imprecise. Although the study design of the concerned studies did not allow proper confirmation that statin intake was continued during hospital admission for COVID-19, it may be safe to assume that statin intake was not discontinued during hospitalization, since there was no directive or recommendation from any clinical guidelines to discontinue statins upon acquisition of COVID-19, despite speculations that statins may cause harms in patients with COVID-19; conversely, continued use is recommended in patients already receiving statins prior to the acquisition of COVID-19, since the established clinical benefits with the use of statins outweigh the possibility of harms in this patient population [3, 61]. In addition, we have now performed a senstivity analyses with studies that confirmed the use of statins during the hospitalization of COVID-19, and the findings are consistent with the main analyses.

Katsiki et al. [8] provided mechanisms that could explain the beneficial impact of statins in patients with COVID19 , other than those we have described in our previous

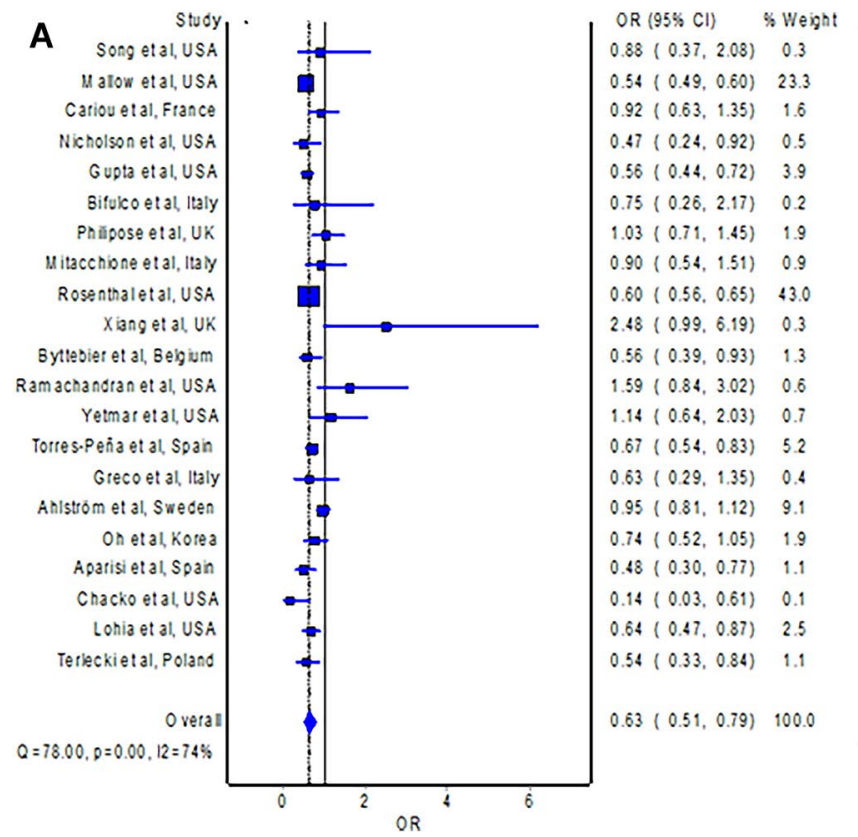

Fig. 2 A Forest plot showing the pooled OR of mortality between statin users and non-statin users with COVID-19. B Forest plot showing the pooled HR of mortality between statin users and non-statin users

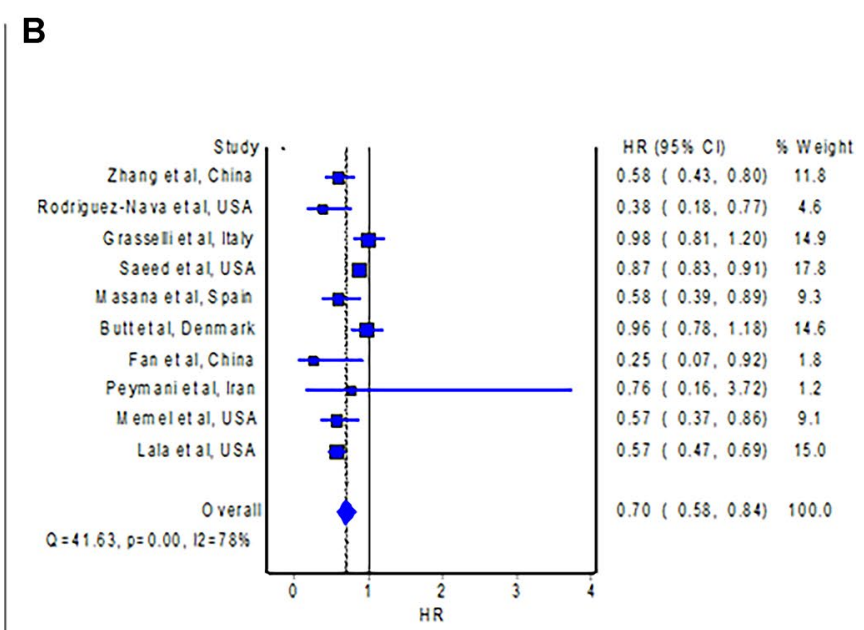

with COVID-19. CI confidence interval, COVID-19 coronavirus disease 2019, $H R$ hazard ratio, $O R$ odds ratio 
Fig. 3 A Forest plot showing the pooled OR of severe illness between statin users and nonstatin users with COVID-19. B Forest plot showing the pooled HR of severe illness between statin users and non-statin users with COVID-19. $C I$ confidence interval, COVID-19 coronavirus disease $2019, H R$ hazard ratio, $O R$ odds ratio
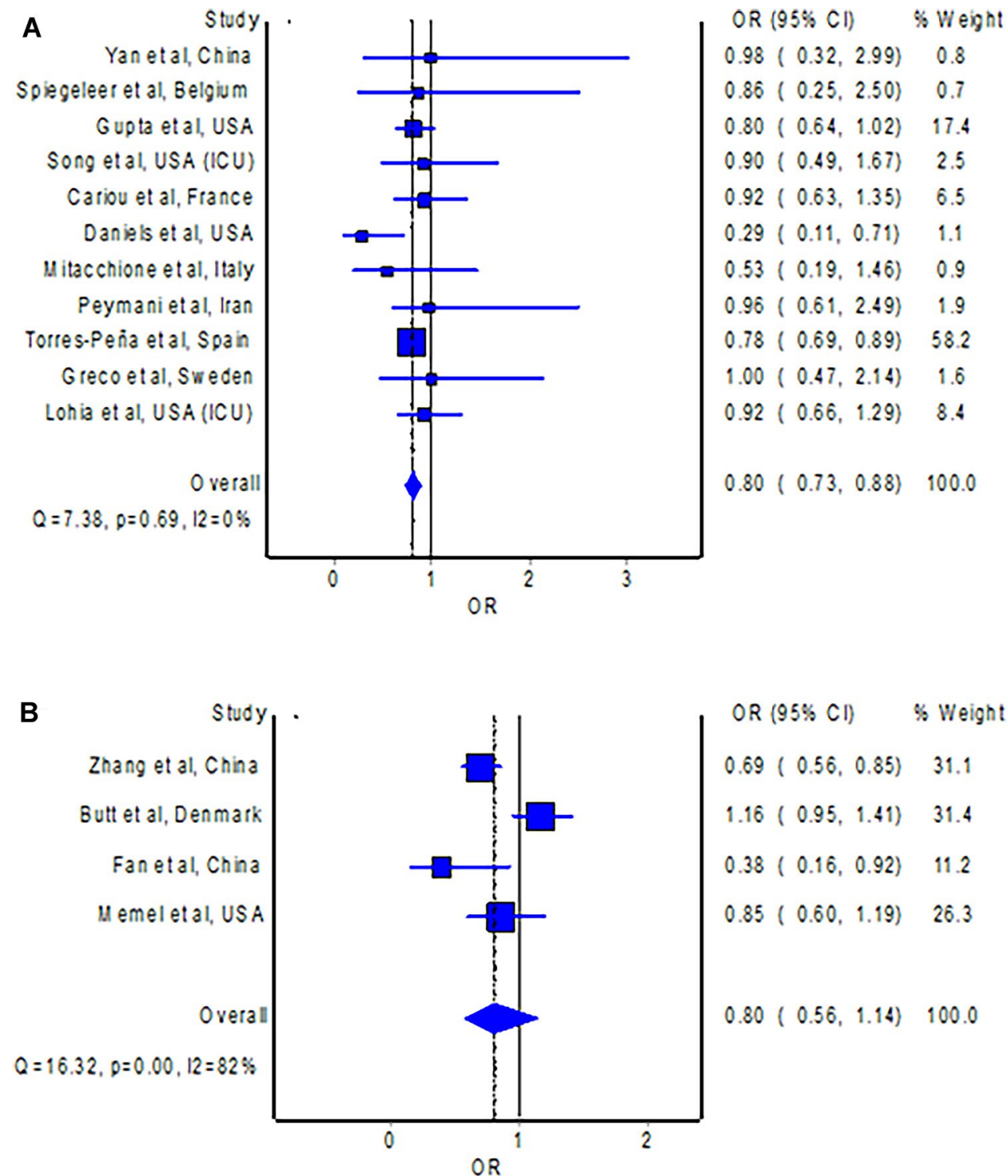

meta-analysis. They hypothesized that statins could exert a protective effect against developing acute kidney injury and acute cardiac injury in patients with COVID-19, both of which are also predictors of mortality in this patient population. We agreed with their proposed mechanism, though we would caution that such an effect has yet to be conclusively proven. In the study by Zhang et al. [12], there was no significant protective effect of statins against the development of both acute kidney injury ( $\mathrm{aHR}=0.72 ; 95 \% \mathrm{CI} 0.51-1.01$ ) and acute cardiac injury ( $\mathrm{aHR}=1.16$; 95\% CI 0.98-1.37). More evaluation from other studies is needed. Katsiki et al. [8] also suggested caution regarding drug-drug interactions between statins and other pharmacological agents used for the treatment of COVID-19, specifically azithromycin and ritonavir/lopinavir. However, the use of both azithromycin and ritonavir/lopinavir in patients with COVID-19 is out of favor currently since available randomized trials have failed to show their benefits in patients with COVID-19 [4, 62-64].

Ganjali et al. [9] have commented that the findings in our previous meta-analysis were in contrast to another metaanalysis by Hariyanto and Kurniawan [65], which reported that the use of statins failed to improve clinical outcomes of hospitalized patients with COVID-19. However, it should be noted that the meta-analysis by Hariyanto and Kurniawan [65] pooled ORs which were estimated directly from crude event numbers without consideration of potential confounders that might modify the association between the use of statins and mortality as well as the development of severe outcomes in patients with COVID-19. In contrast, we extracted and pooled only adjusted measures of effect, which increased the reliability of our findings, and demonstrated clinical benefits using statins in patients with COVID-19. 

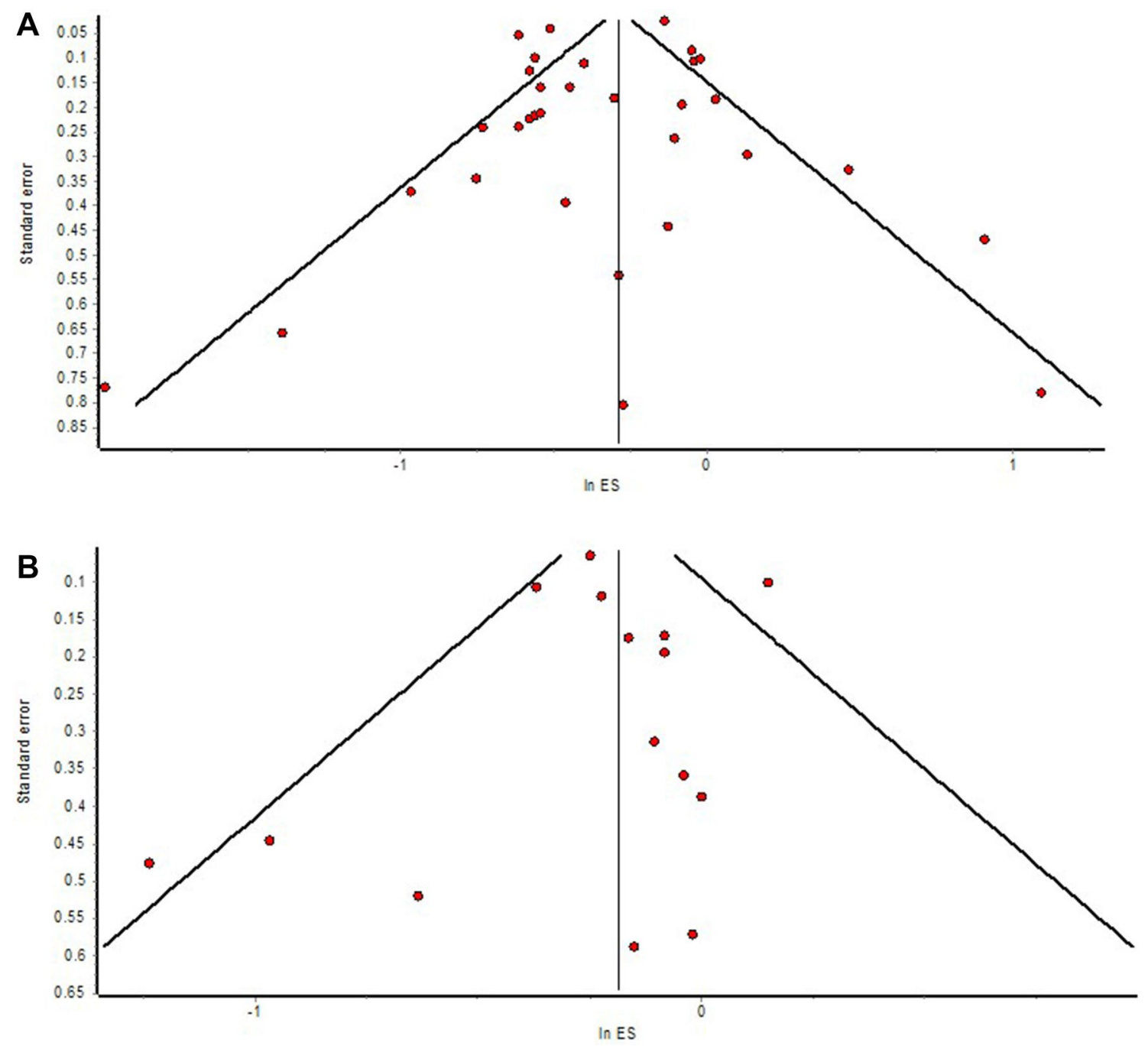

Fig. 4 A Funnel plot of publication bias with studies reporting outcomes on mortality. B Funnel plot of publication bias with studies reporting outcomes on severe illness

Our meta-analysis has several limitations. First, the included studies are retrospective/prospective observational studies. Although studies with retrospective/prospective designs may not be ideal for investigating the causal relationships between interventions and outcomes, adjustment of potential confounders in the respective studies may reduce the risk of bias in the measures of association. Second, it is unclear if statin use was continued during hospitalization for COVID-19 in most of the included studies, but continued use could be safely assumed since there has been no recommendation for discontinuation of statins in patients with COVID-19 thus far. In addition, we have performed sensitivity analyses with studies $[12,13,18,20,27,32,35,36$, $39,42,44]$ that confirmed the continuation of statins during hospitalization for COVID-19. Third, the regimen of statins used was not mentioned in most of the included studies, but based upon studies that provided such information, statin therapy of moderate-to-high intensity could be effective.

\section{Conclusion}

Thus far, there is adequate evidence from observational studies, at the current sample size, to suggest that the use of statins was associated with a significantly reduced risk of development of a fatal course or a severe course of illness in patients with COVID-19. Our findings provide a rationale to investigate the use of statins among patients with COVID19 in large-scale clinical trials. In the meantime, we await more data from prospective studies or randomized controlled trials to substantiate our findings of clinical benefits using statins in patients with COVID-19. In addition, future studies should segregate the analysis based on different types of 
statins (such as lipophilic statins versus hydrophilic statins) to determine if the clinical benefits represent a class effect or if only certain type(s) of statins demonstrates clinical benefits in patients with COVID-19.

\section{Declarations}

Funding No external funding was used in this work.

Conflict of interest Chia Siang Kow and Syed Shazad Hasan declare that they have no known competing financial interests or personal relationships that could have appeared to influence the work reported in this paper.

Ethics approval Not applicable.

Consent to participate Not applicable.

Consent for publication Not applicable.

Availability of data and materials All data generated or analyzed during this study are included in this published article (and its supplementary information files)

Code availability Not applicable.

\section{Reference}

1. Kow CS, Hasan SS. Meta-analysis of effect of statins in patients with COVID-19. Am J Cardiol. 2020;134:153-5.

2. Castiglione V, Chiriacò M, Emdin M, Taddei S, Vergaro G. Statin therapy in COVID-19 infection. Eur Heart J Cardiovasc Pharmacother. 2020;6(4):258-9.

3. Kow CS, Thiruchelvam K, Hasan SS. Pharmacotherapeutic considerations for the management of cardiovascular diseases among hospitalized COVID-19 patients. Expert Rev Cardiovasc Ther. 2020;18(8):475-85.

4. WHO Solidarity Trial Consortium, Pan H, Peto R, et al. Repurposed Antiviral Drugs for Covid-19 - Interim WHO Solidarity Trial Results. N Engl J Med. 2021;384(6):497-511.

5. WHO Rapid Evidence Appraisal for COVID-19 Therapies (REACT) Working Group, Sterne JAC, Murthy S, et al. Association between administration of systemic corticosteroids and mortality among critically ill patients with COVID-19: a Metaanalysis. JAMA. 2020;324(13):1-13.

6. Weintraub WS. Perspective on trends in statin use. JAMA Cardiol. 2017;2(1):11-2.

7. Fedson DS. Statin Treatment of COVID-19. Am J Cardiol. 2020;136:171-3.

8. Katsiki N, Banach M, Mikhailidis DP. More good news on statins and COVID-19. Am J Cardiol. 2020;138:127-8.

9. Ganjali S, Bianconi V, Penson PE, et al. Commentary: Statins, COVID-19, and coronary artery disease: killing two birds with one stone [published online ahead of print, 2020 Sep 23]. Metabolism. 2020;113:154375.

10. Wells G, Shea B, O'Connell D, Peterson J, Welch V, Losos M, Tugwell P. The Newcastle-Ottawa Scale (NOS) for assessing the quality of nonrandomised studies in meta-analyses. 2013. http://www.ohri.ca/programs/clinical_epidemiology/oxford.asp. Accessed 25 Oct 2020.
11. Yan H, Valdes AM, Vijay A, et al. Role of drugs used for chronic disease management on susceptibility and severity of COVID-19: a large case-control study. Clin Pharmacol Ther. 2020;108(6):1185-94.

12. Zhang XJ, Qin JJ, Cheng X, et al. In-hospital use of statins is associated with a reduced risk of mortality among individuals with COVID-19. Cell Metab. 2020;32(2):176-187.e4.

13. Rodriguez-Nava G, Trelles-Garcia DP, Yanez-Bello MA, Chung CW, Trelles-Garcia VP, Friedman HJ. Atorvastatin associated with decreased hazard for death in COVID-19 patients admitted to an ICU: a retrospective cohort study. Crit Care. 2020;24(1):429.

14. Grasselli G, Greco M, Zanella A, et al. Risk factors associated with mortality among patients with COVID-19 in intensive care units in Lombardy, Italy [published online ahead of print, 2020 Jul 15]. JAMA Intern Med. 2020;180(10):1-11.

15. De Spiegeleer A, Bronselaer A, Teo JT, et al. The effects of ARBs, ACEis, and statins on clinical outcomes of COVID-19 infection among nursing home residents. J Am Med Dir Assoc. 2020;21(7):909-14 (e2).

16. Gupta A, Madhavan MV, Poterucha TJ, et al. Association between antecedent statin use and decreased mortality in hospitalized patients with COVID-19. Nat Commun. 2021;12(1):1325.

17. Song SL, Hays SB, Panton CE, et al. Statin use is associated with decreased risk of invasive mechanical ventilation in COVID-19 patients: a preliminary study. Pathogens. 2020;9(9):759.

18. Mallow PJ, Belk KW, Topmiller M, Hooker EA. Outcomes of hospitalized COVID-19 patients by risk factors: results from a United States Hospital Claims Database. J Health Econ Outcomes Res. 2020;7(2):165-74.

19. Cariou B, Goronflot T, Rimbert A, et al. Routine use of statins and increased COVID-19 related mortality in inpatients with type 2 diabetes: Results from the CORONADO study. Diabetes Metab. 2021;47(2):101202.

20. Saeed O, Castagna F, Agalliu I, et al. Statin Use and In-Hospital Mortality in Patients With Diabetes Mellitus and COVID-19. J Am Heart Assoc. 2020;9(24):e018475.

21. Nicholson CJ, Wooster L, Sigurslid HH, et al. Estimating risk of mechanical ventilation and in-hospital mortality among adult COVID-19 patients admitted to Mass General Brigham: the VICE and DICE scores. EClinicalMedicine. 2021;33:100765.

22. Daniels LB, Sitapati AM, Zhang J, et al. Relation of statin use prior to admission to severity and recovery among COVID-19 inpatients. Am J Cardiol. 2020;136:149-55.

23. Bifulco M, Ciccarelli M, Bruzzese D, et al. The benefit of statins in SARS-CoV-2 patients: further metabolic and prospective clinical studies are needed. Endocrine. 2021;71(2):270-2.

24. Masana L, Correig E, Rodríguez-Borjabad C, et al. Effect of statin therapy on SARS-CoV-2 infection-related mortality in hospitalized patients [published online ahead of print, 2020 Nov 2]. Eur Heart J Cardiovasc Pharmacother. 2020;pvaa128.

25. Philipose Z, Smati N, Wong CSJ, et al. Obesity, old age, and frailty are the true risk factors for COVID-19 mortality and not chronic disease or ethnicity. Preprint. medRxiv. 2020.

26. Butt JH, Gerds TA, Schou M, et al. Association between statin use and outcomes in patients with coronavirus disease 2019 (COVID19): a nationwide cohort study. BMJ Open. 2020;10(12):e044421.

27. Fan Y, Guo T, Yan F, et al. Association of statin use with the in-hospital outcomes of 2019-coronavirus disease patients: a retrospective study. Front Med (Lausanne). 2020;7:584870.

28. Mitacchione G, Schiavone M, Curnis A, et al. Impact of prior statin use on clinical outcomes in COVID-19 patients: data from tertiary referral hospitals during COVID-19 pandemic in Italy. J Clin Lipidol. 2021;15(1):68-78.

29. Rosenthal N, Cao Z, Gundrum J, Sianis J, Safo S. Risk factors associated with in-hospital mortality in a US National 
sample of patients with COVID-19. JAMA Netw Open. 2020;3(12):e2029058.

30. Xiang Y, Wong KCY, Hon-Cheong S. Exploring drugs and vaccines associated with altered risks and severity of COVID-19: a UK Biobank cohort study of all ATC level-4 drug categories. Preprint. medRxiv. 2020.

31. Peymani P, Dehesh T, Aligolighasemabadi F, et al. Statins in patients with COVID-19: a retrospective cohort study in Iranian COVID-19 patients. Transl Med Commun. 2021;6(1):3.

32. Byttebier G, Belmans L, Alexander M, et al. Hospital mortality in COVID-19 patients in Belgium treated with statins, ACE inhibitors and/or ARBs [published online ahead of print, 2021 May 28]. Hum Vaccin Immunother. 2021;1-10.

33. Ramachandran P, Perisetti A, Gajendran M, et al. Pre-hospitalization proton pump inhibitor use and clinical outcomes in COVID19 [published online ahead of print, 2020 Nov 30]. Eur J Gastroenterol Hepatol. 2020. https://doi.org/10.1097/MEG.0000000000 002013.

34. Yetmar ZA, Challener DW, Tleyjeh IM, et al. Association between chronic statin use and 30-day mortality in hospitalized patients with COVID-19. Mayo Clin Proc Innov Qual Outcomes. 2021;5(2):442-6.

35. Torres-Peña JD, Pérez-Belmonte LM, Fuentes-Jiménez F, et al. Prior treatment with statins is associated with improved outcomes of patients with COVID-19: data from the SEMI-COVID-19 Registry. Drugs. 2021;81(6):685-95.

36. Memel ZN, Lee JJ, Foulkes AS, Chung RT, Thaweethai T, Bloom PP. Statins are associated with improved 28-day mortality in patients hospitalized with SARS-CoV-2 infection. Preprint. medRxiv. 2021.

37. Greco S, D'Amuri A, Giorgini E, et al. Role of statins in coronavirus-related disease (COVID-19): a retrospective cohort study in Northern Italy [published online ahead of print, 2021 Apr 27]. High Blood Press Cardiovasc Prev. 2021;1-10.

38. Ahlström B, Frithiof R, Hultström M, Larsson IM, Strandberg G, Lipcsey M. The Swedish COVID-19 intensive care cohort: Risk factors of ICU admission and ICU mortality [published online ahead of print, 2021 Jan 12]. Acta Anaesthesiol Scand. 2021;65(4):525-33.

39. Oh TK, Song IA, Jeon YT. Statin therapy and the risk of COVID19: a cohort study of the National Health Insurance Service in South Korea. J Pers Med. 2021;11(2):116.

40. Aparisi Á, Amat-Santos IJ, López Otero D, Marcos-Mangas M, González-Juanatey JR, San Román JA. Impact of statins in patients with COVID-19. Rev Esp Cardiol (Engl Ed). 2021;74(7):637-40.

41. Chacko SR, DeJoy R 3rd, Lo KB, et al. Association of Pre-Admission Statin Use With Reduced In-Hospital Mortality in COVID19. Am J Med Sci. 2021;361(6):725-30.

42. Luo P, Qiu L, Liu Y, et al. Metformin treatment was associated with decreased mortality in COVID-19 patients with diabetes in a retrospective analysis. Am J Trop Med Hyg. 2020;103(1):69-72.

43. Lohia P, Kapur S, Benjaram S, Mir T. Association between antecedent statin use and severe disease outcomes in COVID-19: a retrospective study with propensity score matching. J Clin Lipidol. 2021;15(3):451-9.

44. Terlecki M, Wojciechowska W, Klocek M, et al. Association between cardiovascular disease, cardiovascular drug therapy, and in-hospital outcomes in patients with COVID-19: data from a large single-center registry in Poland. Kardiol Pol. 2021. https:// doi.org/10.33963/KP.15990.

45. Lala A, Johnson KW, Januzzi JL, et al. Prevalence and Impact of myocardial injury in patients hospitalized with COVID-19 infection. J Am Coll Cardiol. 2020;76(5):533-46.
46. Kow CS, Hasan SS. Use of statins in patients with COVID-19. QJM. 2020;113(8):604-5.

47. Lee KCH, Sewa DW, Phua GC. Potential role of statins in COVID19. Int J Infect Dis. 2020;96:615-7.

48. Shresta S. Statin drug therapy may increase COVID-19 infection. Nepal Med J. 2020;5(3):326-7.

49. Goldstein MR, Poland GA, Graeber CW. Are certain drugs associated with enhanced mortality in COVID-19? QJM. 2020;113(7):509-10.

50. Dashti-Khavidaki S, Khalili H. Considerations for statin therapy in patients with COVID-19. Pharmacotherapy. 2020;40(5):484-6.

51. Schönbeck U, Libby P. Inflammation, immunity, and HMG-CoA reductase inhibitors: statins as antiinflammatory agents? Circulation. 2004;109(21 Suppl 1):II18-26.

52. Ortego M, Bustos C, Hernández-Presa MA, et al. Atorvastatin reduces NF-kappaB activation and chemokine expression in vascular smooth muscle cells and mononuclear cells. Atherosclerosis. 1999;147(2):253-61.

53. Gorabi AM, Kiaie N, Bianconi V, et al. Antiviral effects of statins. Prog Lipid Res. 2020;79:101054.

54. Mehrbod P, Omar AR, Hair-Bejo M, Haghani A, Ideris A. Mechanisms of action and efficacy of statins against influenza. Biomed Res Int. 2014;2014:872370.

55. Arslan F, Pasterkamp G, de Kleijn DP. Unraveling pleiotropic effects of statins: bit by bit, a slow case with perspective. Circ Res. 2008;103(4):334-6.

56. Gavriilaki E, Anyfanti P, Gavriilaki M, Lazaridis A, Douma S, Gkaliagkousi E. Endothelial dysfunction in COVID-19: lessons learned from coronaviruses. Curr Hypertens Rep. 2020;22(9):63.

57. Tobert JA. Efficacy and long-term adverse effect pattern of lovastatin. Am J Cardiol. 1988;62(15):28J-34J.

58. Dujovne CA, Chremos AN, Pool JL, et al. Expanded clinical evaluation of lovastatin (EXCEL) study results: IV. Additional perspectives on the tolerability of lovastatin. Am J Med. 1991;91(1B):25S-30S.

59. Boccuzzi SJ, Bocanegra TS, Walker JF, Shapiro DR, Keegan ME. Long-term safety and efficacy profile of simvastatin. Am J Cardiol. 1991;68(11):1127-31.

60. Pedersen TR, Berg K, Cook TJ, et al. Safety and tolerability of cholesterol lowering with simvastatin during 5 years in the Scandinavian Simvastatin Survival Study. Arch Intern Med. 1996;156(18):2085-92.

61. Graham DJ, Staffa JA, Shatin D, et al. Incidence of hospitalized rhabdomyolysis in patients treated with lipid-lowering drugs. JAMA. 2004;292(21):2585-90.

62. Kim AY, Gandhi RT. Coronavirus disease 2019 (COVID-19): Management in hospitalized adults. In: Post TW editor. Waltham: UpToDate Inc.

63. Fiolet T, Guihur A, Rebeaud ME, Mulot M, Peiffer-Smadja N, Mahamat-Saleh Y. Effect of hydroxychloroquine with or without azithromycin on the mortality of coronavirus disease 2019 (COVID-19) patients: a systematic review and meta-analysis. Clin Microbiol Infect. 2021;27(1):19-27.

64. Kow CS, Hasan SS. Azithromycin in patients with COVID-19: Friend or foe? Clin Microbiol Infect. 2021;27(1):136-7.

65. Hariyanto TI, Kurniawan A. Statin therapy did not improve the in-hospital outcome of coronavirus disease 2019 (COVID-19) infection [published online ahead of print, 2020 Aug 26]. Diabetes Metab Syndr. 2020;14(6):1613-5. 